\title{
Drag of electrons in graphene by substrate surface polar phonons
}

\author{
S. V. Koniakhin ${ }^{1,2, \text { 田 and A. V. Nalitov }}{ }^{3}$ \\ ${ }^{1}$ Ioffe Physical-Technical Institute of the Russian Academy of Sciences, 194021 St. Petersburg, Russia \\ ${ }^{2}$ St. Petersburg Academic University - Nanotechnology Research and Education \\ Centre of the Russian Academy of Sciences, 194021 St. Petersburg, Russia \\ ${ }^{3}$ School of Physics and Astronomy, University of Southampton, Southampton SO17 1BJ, United Kingdom
}

\begin{abstract}
It is known that electron scattering by surface polar phonons (SPPs) of the substrate reduces their mobility in supported graphene. However, there is no experimental evidence for contribution of drag of electrons by SPP to thermoelectric phenomena in graphene: graphene thermopower exhibits good agreement with Mott's law, which means that the diffusion contribution to the thermopower is dominant in a wide range of carrier densities and temperatures. Here we develop a complete theory of drag of electrons in graphene by SPP. By solving Boltzmann transport equation for electrons scattered by SPPs we derive SPP drag contribution to the thermopower in graphene. Compared to diffusion thermopower, obtained values appear to be one order of magnitude lower for various substrates. This can be explained by low occupation number of the SPPs and short mean free path of such phonons stemming from their small group velocity. We conclude that experiments on thermopower in graphene can be treated within the framework of Mott's law.
\end{abstract}

\section{INTRODUCTION}

During the past decade there have been intense studies of mechanical ${ }^{12}$, electronic ${ }^{3 / 5}$, optical ${ }^{6 / 8}$, therma ${ }^{9} 11$ and magnetic properties of graphene. Significant role among them plays experimenta 12 , 16 and theoretica $\sqrt{17} \sqrt{24}$ investigation of thermoelectricity. Intriguing thermoelectric phenomena that contributes to thermopower in graphene, as well as in metals ${ }^{25}$, carbon nanocomposites ${ }^{26}$ and graphite ${ }^{27} \sqrt{29}$, is the effect of phonon drage

Suspending graphene sheets at a distance of hundreds of nanometers from the substrate gives possibility to investigate native properties of graphene. However, gating the graphene devices and controlling carriers density requires close contact between graphene sheet and the substrate. Therefore various aspects of graphenesubstrate interaction are of active current research 30 33. Substrate reduces mobility of carriers in graphene due to electron scattering on surface charged impurities ${ }^{5134}$, surface corrugations $\sqrt{357}$ and atomic steps 38 . Molecular dynamics simulations show that van der Walls interaction between graphene ${ }^{39}$ or nanotubes ${ }^{40}$ and substrate significantly reduces relaxation time of intrinsic phonons.

In recent studies it was shown that scattering by surface polar phonons (SPPs) in substrates like $\mathrm{SiO}_{2}$ and $\mathrm{SiC}$ reduce electron mobility in graphene $\mathrm{41}^{44}$ and carbon nanotubes 45/46. However, current experimental data on thermoelectric properties of supported graphene 12 show that behavior of thermopower in graphene coincides with Mott law that describes the diffusion contribution. Therefore is important to elucidate why the contribution from drag by substrate phonons have not been robustly detected yet.

In this paper we consider a monolayer graphene with linear electron dispersion law $\varepsilon_{\mathbf{k}}=\hbar v_{F} k$, and degenerate electron gas obeying Fermi statistics. Fermi energy is related to the carrier density by $\varepsilon_{F}=\hbar v_{F} k_{F}=\hbar v_{F} \sqrt{\pi n}$. It is useful to introduce the dimensionless electron wave vector $\tilde{k}=k a_{0} / \pi$. The tilde is used to denote other quantities, normalized in the same manner.

\section{THEORY}

Surface polar phonons of the substrate generate an electric field at significant distances from the substrate (see fig. 1 from ref ${ }^{45}$ ). The phonon-induced field penetrates graphene at the surface of the substrate and provides the probability for an electron in graphehe to be scattered by remote substrate phonon. Electron scattering by SPP is not the dominant mechanism of electron mobility reduce in relatively thick semiconductor layers in metal-oxide-semiconductor field-effect transistors, but plays significant role for graphene and carbon nanotubes.

The electron transition rate arising from scattering by SPPs is given by 42

$$
W_{\mathbf{k} \rightarrow \mathbf{k}+\mathbf{q}}^{S P P}=A_{\mathbf{k}, \mathbf{q}} \frac{4 \pi^{2} e^{2} F^{2}}{S q} \exp \left(-2 q z_{0}\right),
$$

where $\mathbf{k}$ and $\mathbf{q}$ are electron and phonon wave vectors respectively, $e$ is the electron charge, $S$ is a surface of graphene sheet and $z_{0} \approx 3.5 \AA$ is the van der Waals distance between graphene sheet and substrate. Multiplier $A_{\mathbf{k}, \mathbf{q}}=\frac{1}{2}\left(1+\cos \left(\theta_{\mathbf{k}+\mathbf{q}}-\theta_{\mathbf{k}}\right)\right)$ arises from the chiral nature of carriers in graphene. The electric field magnitude and consequently the scattering rate $W$ overall are defined by Frölich coupling

$$
F^{2}=\frac{\hbar \omega_{p h}}{2 \pi}\left(\frac{1}{\varepsilon_{\infty}+\varepsilon_{e n v}}-\frac{1}{\varepsilon_{0}+\varepsilon_{e n v}}\right)
$$

where $\varepsilon_{\infty}$ and $\varepsilon_{0}$ are low- and high-frequency dielectric constants of the substrate and $\varepsilon_{e n v}$ is the environment dielectric constant. Following ref ${ }^{\frac{142}{2}}$ we assume the latter to be equal to 1 . As it can be seen from exponential 
multiplier in (1), $q^{-1}$ is a characteristic distance at which the electric field decays outside the substrate. In table 2 of ref. 42 the energies of surface optical phonons and values of Frölich coupling strength for various substrate materials are listed. For variety of substrates $\hbar \omega_{p h} \sim$
$0.1 \mathrm{eV}$ and $F^{2}$ value is close to $0.5 \mathrm{meV}$.

The SPP phonon collision integral, entering the Boltzmann transport equation on the electron distribution function $f\left(\varepsilon_{\mathbf{k}}\right)$, can be written down as

$$
\begin{aligned}
\left(\frac{\partial}{\partial t} f(k)\right)_{p h}=-\frac{2 \pi}{\hbar} & \frac{S}{4 \pi^{2}} \int d \mathbf{q} \times \\
\quad & \quad W_{k \rightarrow k+q} N_{p h}(q) f^{(0)}\left(\varepsilon_{k}\right)\left(1-f^{(0)}\left(\varepsilon_{k+q}\right)\right) \delta\left(\varepsilon_{k}-\varepsilon_{k+q}-\hbar \omega_{p h}\right)+ \\
& W_{k \rightarrow k-q} f^{(0)}\left(N_{p h}(q)+1\right) f^{(0)}\left(\varepsilon_{k}\right)\left(1-f^{(0)}\left(\varepsilon_{k-q}\right)\right) \delta\left(\varepsilon_{k}-\varepsilon_{k-q}+\hbar \omega_{p h}\right) \\
& -W_{k-q \rightarrow k} N_{p h}(q) f^{(0)}\left(\varepsilon_{k-q}\right)\left(1-f^{(0)}\left(\varepsilon_{k}\right)\right) \delta\left(\varepsilon_{k}-\varepsilon_{k-q}+\hbar \omega_{p h}\right)- \\
& \left.W_{k+q \rightarrow k}\left(N_{p h}(q)+1\right) f^{(0)}\left(\varepsilon_{k+q}\right)\left(1-f^{(0)}\left(\varepsilon_{k}\right)\right) \delta\left(\varepsilon_{k}-\varepsilon_{k+q}-\hbar \omega_{p h}\right)\right]
\end{aligned}
$$

where $f^{(0)}\left(\varepsilon_{\mathbf{k}}\right)$ is the equilibrium Fermi distribution function.

In presence of temperature gradient phonon distribution function $N_{p h}(q)$ writes as

$$
N_{p h}(\mathbf{q})=N_{p h}^{(0)}(q)+N_{p h}^{(1)}(\mathbf{q})
$$

In the equation above $N_{p h}^{(0)}(q)$ is the Bose equilibrium distribution function for phonons and $N_{p h}^{(1)}(\mathbf{q})$ is a small correction due to the temperature gradient, which can be written in the relaxation time approximation as

$$
N_{p h}^{(1)}(\mathbf{q})=\tau_{p h}(q) \mathbf{v}(\mathbf{q}) \vec{\nabla} T \frac{\partial N_{p h}^{(0)}(q)}{\partial T}
$$

where $\mathbf{v}(\mathbf{q})=\nabla_{\mathbf{q}} \omega_{p h}$ is a group velocity of optical phonons. The dispersion law of optical phonons writes as

$$
\omega_{p h}(q)=\omega_{0}\left(1-\beta \tilde{q}^{2}\right),
$$

where $\tilde{q}=q a_{0} / \pi$ is the phonon wave vector of normalized to graphene lattice constant $a_{0}=2.46 \AA$. Approximating optical phonons dispersion curves of many semiconductors and dielectrics allows one to estimate $\beta \sim 0.1$.

We assume that the optical phonon lifetime $\tau_{p h}$ is independent on the phonon wave vector $q$. Multiple studies devoted to molecular dynamics simulations $47-50$ and Raman experiments $51+56$ indicate optical phonon lifetime of about dozens of picoseconds in various solids, and we set $\tau_{p h}=10 \mathrm{ps}$. The optical phonon group velocity is linear in $q$, therefore $\tau_{p h} v(q)=L_{0} \tilde{q}$, where $L_{0}=2 \tau_{p h} \omega_{p h} \beta \frac{a_{0}}{\pi}$. Taking $\tau_{p h}=10 \mathrm{ps}, \hbar \omega_{p h}=0.1 \mathrm{eV}$ and $\beta=0.1$ yields $L_{0}=25 \mathrm{~nm}$.

Electrons in graphene are scattered by phonons with $q$ of the order of $k_{F} \ll \pi / a_{0}$ and for actual values of Fermi energy $\hbar \omega_{p h} \beta \tilde{k}_{F}^{2} \ll k_{B} T$. Thus the occupation number of phonons does not depend significantly on magnitude of the phonon wave vector $q$ and the quantity

$$
\frac{\partial N_{p h}^{(0)}(q)}{\partial T}=k_{B} \frac{\hbar \omega_{p h} e^{\frac{\hbar \omega_{p h}}{k_{B} T}}}{\left(e^{\frac{\hbar \omega_{p h}}{k_{B} T}}-1\right)^{2}\left(k_{B} T\right)^{2}}
$$

is considered below to be constant.

When $N_{p h}^{(0)}$ is substituted to the right-hand part of (3), the latter vanishes due to energy conservation law entering the delta functions. After replacing $\mathbf{q}$ with $-\mathbf{q}$ in the second and the third terms in the square brackets in (3), substituting first correction for phonon distribution (5), one obtains the following correction for the electron distribution function within the framework of relaxation time approximation:

$$
f^{(1)}(\mathbf{k})=-\frac{2 \pi \tau(k)}{v_{F}} \frac{e^{2} F^{2}}{\hbar^{2}} L_{0} \frac{\partial N_{p h}^{(0)}(q)}{\partial T}\left(\frac{\mathbf{k}}{k} \vec{\nabla} T\right) I_{1}(\tilde{k})
$$

where $\tau(k)$ is the electron relaxation time and $I_{1}(\tilde{k})$ is the dimensionless integral over phonon wave vector:

$$
\begin{gathered}
I_{1}(\tilde{k})=\int_{0}^{1} d \tilde{q} d \theta \tilde{q} A_{\mathbf{k}, \mathbf{q}} \exp ^{-2 z_{0} \tilde{q} \frac{\pi}{a_{0}}} \cos (\theta) \times \\
\left(f^{(0)}(\tilde{k})-f^{(0)}(|\tilde{\mathbf{k}}+\tilde{\mathbf{q}}|)\right) \times \\
{\left[\delta\left(\tilde{k}+\tilde{\omega}_{p h}-|\tilde{\mathbf{k}}+\tilde{\mathbf{q}}|\right)-\delta\left(\tilde{k}-\tilde{\omega}_{p h}-|\tilde{\mathbf{k}}+\tilde{\mathbf{q}}|\right)\right],}
\end{gathered}
$$

where $\tilde{\omega}_{p h}=\frac{\omega_{p h} a_{0}}{v_{F} \pi}, \tilde{\mathbf{k}}=\frac{\mathbf{k} a_{0}}{\pi}$ and $f^{(0)}(\tilde{k})=(1+$ $\left.\exp \left(\left(\pi \hbar v_{f} \tilde{k} / a_{0}\right)-\varepsilon_{F}\right) / k_{B} T\right)^{-1}$. Fig. 1(a) shows a typical profile of the integral $I_{1}(\tilde{k})$ as a function of electron wave vector amplitude for various Fermi energies. 
In the case of nearly elastic scattering of electrons by acoustic phonons the expression in the round brackets in (9) yields $\pm \frac{\partial f^{(0)}\left(\varepsilon_{k}\right)}{\partial \varepsilon} \hbar \omega_{p h}(q) \approx \pm \hat{\delta}\left(\varepsilon_{k}-\varepsilon_{F}\right) \hbar \omega_{p h}(q)$, where $\hat{\delta}$ is a delta function with a broadening of the order of temperature.

In contrast with the case of acoustic phonons, here $f^{(1)}$ is not linear in $\frac{\partial f^{(0)}}{\partial \varepsilon}$. However, $f^{(1)}$ differs from zero for wave vectors $k$ close to elastic circle of the radius $k_{F}$ (see. fig. 1(a) . Therefore, in (8) we can assume $\tau(k)=\tau\left(k_{F}\right)=$ const without significant loss of accuracy. Electron conductivity in graphene is directly related to electron transport relaxation time as $\$ \frac{442}{42}$

$$
\sigma=\frac{e^{2}}{\hbar} \frac{v_{F} k_{F} \tau\left(k_{F}\right)}{\pi} .
$$

The SPP driven current is given by integral

$$
j_{p h}=\frac{\pi^{2} e v_{F}}{a_{0}^{2}} \int d \tilde{\mathbf{k}} f^{(1)}(\tilde{\mathbf{k}}) .
$$

Substituting the electron distribution correction (8) and dividing the obtained expression by conductivity 10 yields the SPP-drag thermopower:

$$
S_{\mathrm{SPP}}=\frac{\pi^{4} k_{B}}{e} \frac{F^{2} e^{2}}{\varepsilon_{F} a_{0} k_{B} T} \frac{L_{0}}{a_{0}} \frac{\hbar \omega_{p h}}{k_{B} T} \frac{e^{\frac{\hbar \omega_{p h}}{k_{B} T}}}{\left(e^{\frac{\hbar \omega_{p h}}{k_{B} T}}-1\right)^{2}} \cdot I_{2},
$$

where

$$
I_{2}=\frac{1}{2} \int_{0}^{1} \tilde{k} d \tilde{k} I_{1}(\tilde{k}) .
$$

As usually the expression for the thermopower has a form of $\frac{k_{B}}{e}=86 \mu \mathrm{VK}^{-1}$ times a dimensionless factor, which depends on $\varepsilon_{F}$, $T$ etc.

In Fig. 1(b) we have shown the dependence of $I_{2}$ on the Fermi energy in graphene sample. Due to the fact that $I_{1}(k) \approx \frac{\partial}{\partial \varepsilon} f^{(0)}\left(\varepsilon-\frac{1}{2} \hbar \omega_{p h}\right)$, for actual values of the Fermi energy, temperature and phonon frequency $I_{2}$ was approximated via formula

$$
I_{2} \simeq A_{0}\left(\frac{\varepsilon_{F}+\frac{1}{2} \hbar \omega_{p h}}{\varepsilon_{0}}\right)\left(\frac{\varepsilon_{F}}{\varepsilon_{0}}\right)^{0.7}\left(\frac{\hbar \omega_{p h}}{\varepsilon_{0}}\right)^{0.7}
$$

where $A_{0}=5 \cdot 10^{-6}$ and $\varepsilon_{0}=0.1 \mathrm{eV}$, and sufficient accuracy was reached.

\section{RESULTS AND DISCUSSION}

Table 1 lists the values of the SPP drag thermopower for graphene on various substrates. The values $\varepsilon_{F}=$ $0.5 \mathrm{eV}$ and $T=300 \mathrm{~K}$ were used. Values of phonon energies and magnitudes of Frölich coupling were adopted from 42 .
For $\varepsilon_{F}=0.5 \mathrm{eV}, T=300 \mathrm{~K}$ and phonon lifetime $\tau_{p h}=$ $10 \mathrm{ps}$ one has $S_{\mathrm{SPP}} \approx 2 \mu \mathrm{VK}^{-1}$ for graphene on $\mathrm{SiO}_{2}$ substrate, which is one order of magnitude lower than the diffusion contribution. One sees that the $S_{\mathrm{SPP}}$ is of the same order for other considered substrates.

Substitution of (14) to (12) allows us to conclude that the SPP thermopower moderately grows with Fermi energy in graphene. Due to exponential growth with temperature of the optical phonons occupation number, the SPP drag mechanism is only significant at relatively high temperatures. Fig. 2 shows $S_{\mathrm{SPP}}$ as a function of the Fermi energy and the temperature. By different dependencies on temperature and Fermi energy SPP contribution to the thermopower can be straightly distinguished from the ones of diffusion and intrinsic phonon drag mechanism.

To suggest the substrate with maximal SPP drag effect it is important to analyze how $S_{\mathrm{SPP}}$ depends on the optical phonon energy. Equation (2) shows that Frölich coupling linearly grows with phonon energy and by averaging over dielectric constants of various materials one can write the following approximate relation: $F^{2}=0.005 \hbar \omega_{p h}$. Substituting this to (12) allows to plot $S_{\mathrm{SPP}}$ as a function of energy of the substrate phonon. Fig. 3 shows that the phonon energy most favorable for increasing of SPP drag thermopower is about $0.1 \mathrm{eV}$. The energies of optical surface phonons in considered substrates are close to this value. It means that maximal achievable value of $S_{\mathrm{SPP}}$ is reached for considered substrates.

It is interesting to compare coupling between electrons and intrinsic acoustic phonons with the one for SPPs. The coupling of electrons with intrinsic acoustic phonons in graphene reads as ${ }^{22 \mid 44}$.

$$
W_{\mathbf{k} \rightarrow \mathbf{k}+\mathbf{q}}^{\text {intrinsic }}=A_{\mathbf{k}, \mathbf{q}} \frac{\hbar D^{2} q}{\rho S v_{s}},
$$

where $v_{s}$ is the sound velocity in graphene.

For the phonon wave vector $q$ of the order of Fermi wave vector $k_{F}$ corresponding to $\varepsilon_{F}=0.1 \mathrm{eV}$ one can estimate $W^{S P P} / W^{\text {intrinsic }} \approx 600$ and for $\varepsilon_{F}=0.5 \mathrm{eV}$ one obtains $W^{S P P} / W^{\text {intrinsic }} \approx 15$. It means that electrostatic interaction of electrons with remote substrate phonons can be stronger than coupling with intrinsic phonons via deformation potential.

Nevertheless, the strong coupling between electrons in graphene and SPPs is not enough to overcome exponentially low occupation number of SPPs and short mean free path stemming from low group velocity of phonons with $q \approx k_{F}$. We conclude that the contribution to thermopower from drag of electrons by SPPs is weaker than the diffusion contribution, which explains current experimental data on the thermopower in graphene $e^{12}[15$, being in agreement with Mott's law.

The effect of phonon drag will potentially play a role for a substrate with high optical phonon lifetime. Limitation of the phonon lifetime due to three-phonon processes will not allow dominance of SPP drag contribution in 


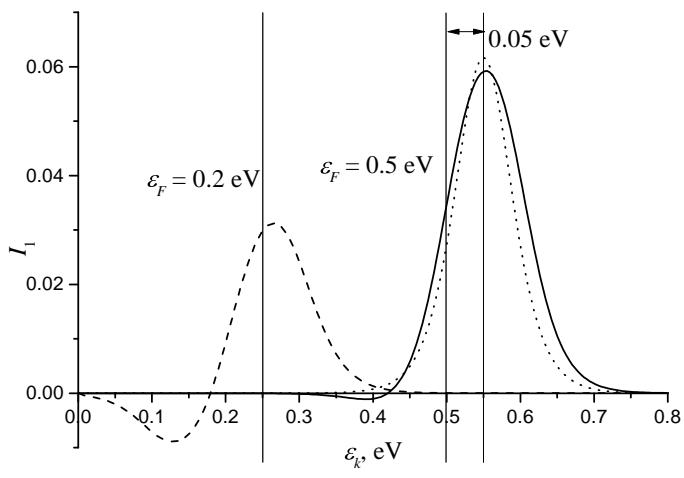

(a)

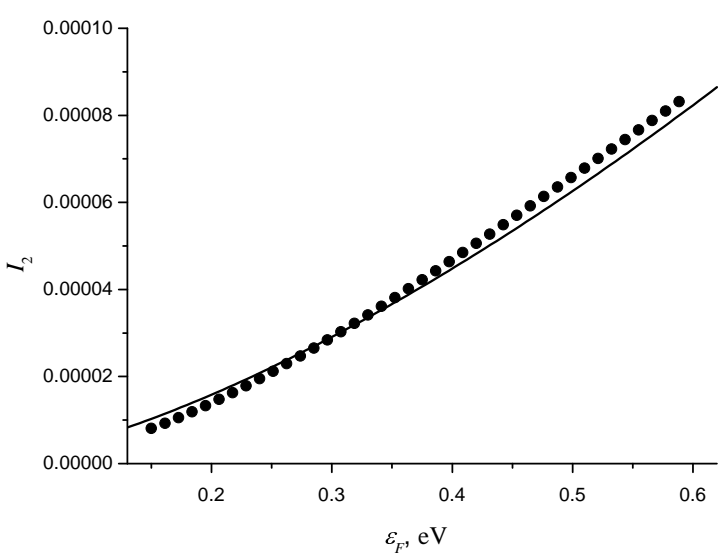

(b)

FIG. 1. Panel (a). Dependence of $I_{1}(\tilde{k})$ magnitude on $\varepsilon_{k}=\hbar v_{F} \pi \tilde{k} / a_{0}$. Optical phonon energy is $0.1 \mathrm{eV}$. Solid curve is for $\varepsilon_{F}=0.5 \mathrm{eV}$ and dashed curve is for $\varepsilon_{F}=0.2 \mathrm{eV}$. Interestingly to notice that for large Fermi energies $I_{1}(k) \approx \frac{\partial}{\partial \varepsilon} f^{(0)}\left(\varepsilon-\frac{1}{2} \hbar \omega_{p h}\right)$, denoted by dotted curve. Panel (b). $I_{2}$ as a function of Fermi energy. $T=300 \mathrm{~K}, \hbar \omega_{p h}=0.1$ eV. Circles denote results of numerical integration and black line curve shows approximation with (14).

TABLE I. SPP drag thermopower in for graphene on $\mathrm{SiO}_{2}, \mathrm{HfO}_{2}, \mathrm{SiC}$ and h-BN. $\varepsilon_{F}=0.5 \mathrm{eV}$ and $\mathrm{T}=300 \mathrm{~K}$. Values are given in $\mu \mathrm{VK}^{-1}$.

\begin{tabular}{lcccr}
\hline \hline & $\mathrm{SiO}_{2}$ & $\mathrm{HfO}_{2}$ & $\mathrm{SiC}$ & $\mathrm{h}-\mathrm{BN}$ \\
\hline const $\tau_{p h}=10 \mathrm{ps}(\mathrm{a}=1)$ & 1.7 & 1.1 & 1.1 & 0.6 \\
const $L_{p h}=1 \mu \mathrm{m}(\mathrm{a}=0)$ & $1.0 \cdot 10^{3}$ & $3 \cdot 10^{3}$ & $6 \cdot 10^{2}$ & $3 \cdot 10^{2}$ \\
\hline \hline
\end{tabular}

graphene thermopower with high probability. Moreover to obtain the predicted values of thermopower the interface between graphene sheet and substrate is to be thin and smooth. In case of mechanically exfoliated graphene the distance between substrate and graphene sheet can be expected to be higher than adopted here value. For epitaxial graphene dead buffer layer can also negatively effect on graphene properties 57 .

The considered effect of SPP drag can be expected even for nonpolar substrates like diamond due to polarizability of interatomic bonds $\sqrt{58}$, which is important for creating composite nanocarbon-based thermoelectric devices. However we one sees that for nonpolar substrates the SPP drag thermopower will be smaller than for polar substrates considered in this paper.

\section{ACKNOWLEDGEMENTS}

This study was supported by Russian Science Foundation (grant \# 16-19-00075). The author is grateful to to E.D. Eidelman, who has encouraged present study. We are gratefully indebted to M.M. Glazov for fruitful discussions.
* kon@mail.ioffe.ru

1 I. Frank, D. M. Tanenbaum, A. Van der Zande, and P. L. McEuen, Journal of Vacuum Science \& Technology B 25, 2558 (2007).

2 C. Lee, X. Wei, J. W. Kysar, and J. Hone, science 321, 385 (2008).

3 A. H. Castro Neto, F. Guinea, N. M. R. Peres, K. S. Novoselov, and A. K. Geim, Rev. Mod. Phys. 81, 109 (2009)

${ }^{4}$ S. Das Sarma, S. Adam, E. H. Hwang, and E. Rossi, Rev. Mod. Phys. 83, 407 (2011)
5 E. H. Hwang, S. Adam, and S. Das Sarma, Phys. Rev. Lett. 98, 186806 (2007).

${ }^{6}$ A. V. Nalitov, L. E. Golub, and E. L. Ivchenko, Phys. Rev. B 86, 115301 (2012).

M. Glazov and S. Ganichev, Physics Reports 535, 101 (2014), high frequency electric field induced nonlinear effects in graphene.

8 J. Karch, C. Drexler, P. Olbrich, M. Fehrenbacher, M. Hirmer, M. M. Glazov, S. A. Tarasenko, E. L. Ivchenko, B. Birkner, J. Eroms, D. Weiss, R. Yakimova, S. LaraAvila, S. Kubatkin, M. Ostler, T. Seyller, and S. D. 


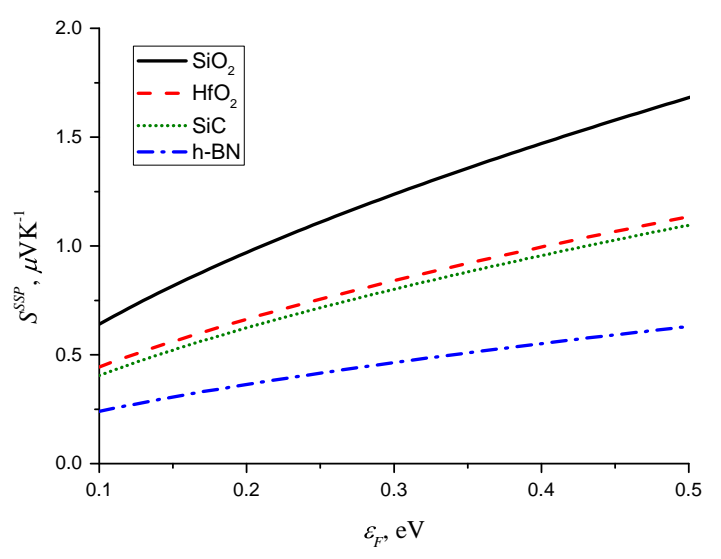

(a)

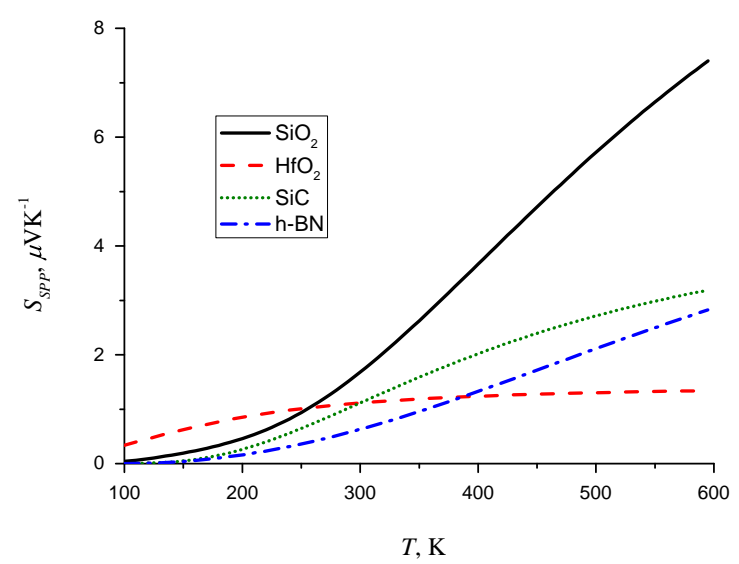

(b)

FIG. 2. SPP drag thermopower in graphene on various substrates. Constant phonon lifetime $\tau_{p h}$ is assumed to be 10 ps. Panel (a). Dependence of $S_{\mathrm{SPP}}$ on Fermi energy in graphene, $T=300 \mathrm{~K}$. Panel (b). Dependence of $S_{\mathrm{SPP}}$ on temperature for constant phonon lifetime, $\varepsilon_{F}=0.5 \mathrm{eV}$.

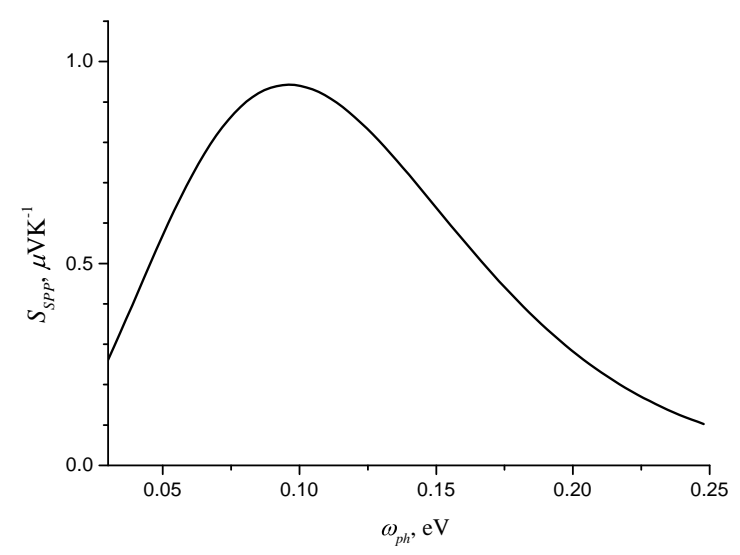

FIG. 3. SPP drag thermopower as a function of phonon energy. $T=300 \mathrm{~K}, \varepsilon_{F}=0.5 \mathrm{eV}$. Phonon lifetime $\tau_{p h}=0.1 \mathrm{ps}$.

Ganichev, Phys. Rev. Lett. 107, 276601 (2011)

9 A. Alofi and G. P. Srivastava, Journal of Applied Physics 112, 013517 (2012)

io D. Nika, E. Pokatilov, A. Askerov, and A. Balandin, Physical Review B 79, 155413 (2009).

11 D. L. Nika and A. A. Balandin, Journal of Physics: Condensed Matter 24, 233203 (2012).

12 J. G. Checkelsky and N. P. Ong, Phys. Rev. B 80, 081413 (2009)

${ }^{13}$ D. Wang and J. Shi, Phys. Rev. B 83, 113403 (2011)

14 Y. M. Zuev, W. Chang, and P. Kim, Phys. Rev. Lett. 102, 096807 (2009).

${ }^{15}$ P. Wei, W. Bao, Y. Pu, C. N. Lau, and J. Shi, Phys. Rev. Lett. 102, 166808 (2009)

${ }^{10}$ P. Dollfus, V. H. Nguyen, et al., Journal of Physics: Condensed Matter 27, 133204 (2015).
17 S. S. Kubakaddi, Phys. Rev. B 79, 075417 (2009)

18 E. H. Hwang, E. Rossi, and S. Das Sarma, Phys. Rev. B 80, 235415 (2009)

19 S. S. Kubakaddi and K. S. Bhargavi, Phys. Rev. B 82, 155410 (2010).

${ }^{20}$ R. G. Vaidya, N. S. Sankeshwar, and B. G. Mulimani, Journal of Applied Physics 112, 093711 (2012).

21 N. S. Sankeshwar, R. G. Vaidya, and B. G. Mulimani, physica status solidi (b) 250, 1356 (2013).

22 S. Koniakhin and E. Eidelman, EPL (Europhysics Letters) 103, 37006 (2013).

23 Z. Alisultanov, Physica E: Low-dimensional Systems and Nanostructures 69, 89 (2015).

24 Z. Alisultanov and N. Mirzegasanova, Technical Physics 59, 1562 (2014).

${ }^{25}$ L. Gurevich, J. Phys. (USSR) 9, 4 (1946).

${ }^{26}$ E. D. Eidelman and A. Y. Vul, Journal of Physics: Condensed Matter 19, 266210 (2007)

27 C. Ayache, A. de Combarieu, and J. P. Jay-Gerin, Phys. Rev. B 21, 2462 (1980).

28 K. Sugihara, Phys. Rev. B 28, 2157 (1983)

29 K. Sugihara, Y. Hishiyama, and A. Ono, Phys. Rev. B 34, 4298 (1986)

so D. R., Y. F., MericI., LeeC., WangL., SorgenfreiS., WatanabeK., TaniguchiT., KimP., S. L., and HoneJ., Nat Nano 5, $722(2010)$.

31 O. Frank, J. Vejpravova, V. Holy, L. Kavan, and M. Kalbac, Carbon 68, 440 (2014).

${ }^{32}$ Y. y. Wang, Z. h. Ni, T. Yu, Z. X. Shen, H. m. Wang, Y. h. Wu, W. Chen, and A. T. Shen Wee, The Journal of Physical Chemistry C 112, 10637 (2008), http://pubs.acs.org/doi/pdf/10.1021/jp8008404

33 R. Brako, D. Sokčević, P. Lazić, and N. Atodiresei, New Journal of Physics 12, 113016 (2010)

${ }^{34}$ T. Ando, Journal of the Physical So$\begin{array}{lllll}\text { ciety of Japan } & \mathbf{7 5}, & 074716 & \text { (2006) }\end{array}$ http://dx.doi.org/10.1143/JPSJ.75.074716

35 M. Ishigami, J. H. Chen, W. G. Cullen, M. S. Fuhrer, and E. D. Williams, Nano Letters 7, 1643 (2007), pMID: 
17497819, http://dx.doi.org/10.1021/nl070613a.

36 S. V. Morozov, K. S. Novoselov, M. I. Katsnelson, F. Schedin, D. C. Elias, J. A. Jaszczak, and A. K. Geim, Phys. Rev. Lett. 100, 016602 (2008)

37 M. Katsnelson and A. Geim, Philosophical Transactions of the Royal Society of London A: Mathematical, Physical and Engineering Sciences 366, 195 (2008), http://rsta.royalsocietypublishing.org/content/366/1863/1

$38 \mathrm{H}$. Sevinli and M. Brandbyge, Applied Physics Letters 105, 153108 (2014), http://dx.doi.org/10.1063/1.4898066

39 B. Qiu and X. Ruan, Applied Physics Letters 100, 193101 (2012), http://dx.doi.org/10.1063/1.4712041

40 Z.-Y. Ong, E. Pop, and J. Shiomi, Phys. Rev. B 84, 165418 (2011)

41 S. Fratini and F. Guinea, Phys. Rev. B 77, 195415 (2008)

${ }^{42}$ V. Perebeinos and P. Avouris, Phys. Rev. B 81, 195442 (2010)

43 J.-H. Chen, C. Jang, S. Xiao, M. Ishigami, and M. S. Fuhrer, Nat Nano 3, 206 (2008).

${ }^{44}$ K. Bhargavi and S. Kubakaddi, Physica E: Lowdimensional Systems and Nanostructures 52, 116 (2013).

${ }^{45}$ V. Perebeinos, S. V. Rotkin, A. G. Petrov, and P. Avouris, Nano Letters 9, 312 (2009), pMID: 19055370, http://dx.doi.org/10.1021/nl8030086.

46 A. G. Petrov and S. V. Rotkin, JETP Letters 84, 156.
47 K. Esfarjani, G. Chen, and H. T. Stokes, Physical Review B 84, 085204 (2011).

${ }^{48}$ Y. He, D. Donadio, and G. Galli, Applied physics letters 98, 144101 (2011).

49 J. Turney, E. Landry, A. McGaughey, and C. Amon, Physical Review B 79, 064301 (2009).

${ }^{50}$ H. Bao, B. Qiu, Y. Zhang, and X. Ruan, Journal of Quanull.pidfitive Spectroscopy and Radiative Transfer 113, 1683 (2012).

51 S. Anand, P. Verma, K. Jain, and S. Abbi, Physica B: Condensed Matter 226, 331 (1996).

52 C. Aku-Leh, J. Zhao, R. Merlin, J. Menendez, and M. Cardona, Physical Review B 71, 205211 (2005).

53 D. Song, F. Wang, G. Dukovic, M. Zheng, E. Semke, L. E. Brus, and T. F. Heinz, Physical review letters 100, 225503 (2008).

54 J. J. Letcher, K. Kang, D. G. Cahill, and D. D. Dlott, Applied physics letters 90, 252104 (2007).

55 M. S. Liu, L. A. Bursill, S. Prawer, and R. Beserman, Phys. Rev. B 61, 3391 (2000)

56 K. Lee, B. J. Sussman, J. Nunn, V. Lorenz, K. Reim, D. Jaksch, I. Walmsley, P. Spizzirri, and S. Prawer, Diamond and Related Materials 19, 1289 (2010).

57 Y. Qi, S. Rhim, G. Sun, M. Weinert, and L. Li, Physical review letters 105, 085502 (2010).

58 G. Mahan, Physical Review B 79, 075408 (2009). 